\title{
Estrés académico y patrón alimentario en estudiantes universitarios de la Licenciatura en Arquitectura
}

Moreno-Jaime Alejandra Fabiola*, Gallegos-González María del Carmen*, Zúñiga-Rentería Óscar Samuel*, Torres-Montoya Roberto*, Serna-Martínez Liliana*, Rodríguez- Moreno Gabriela Selenne*, Martín del Campo-Cervantes Judith**

\begin{tabular}{|c|c|}
\hline 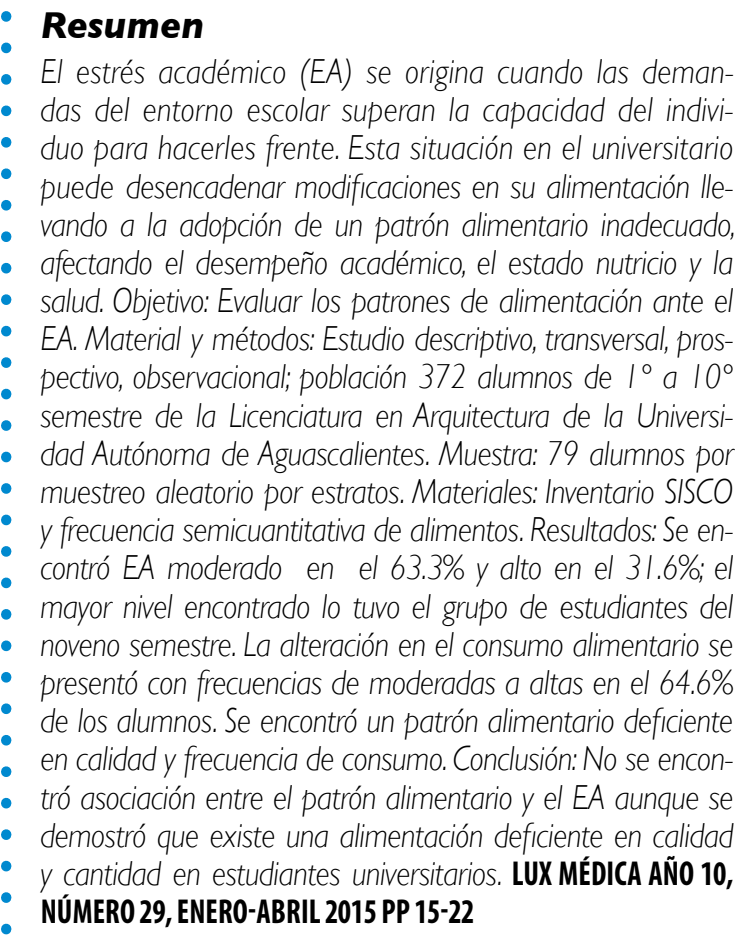 & 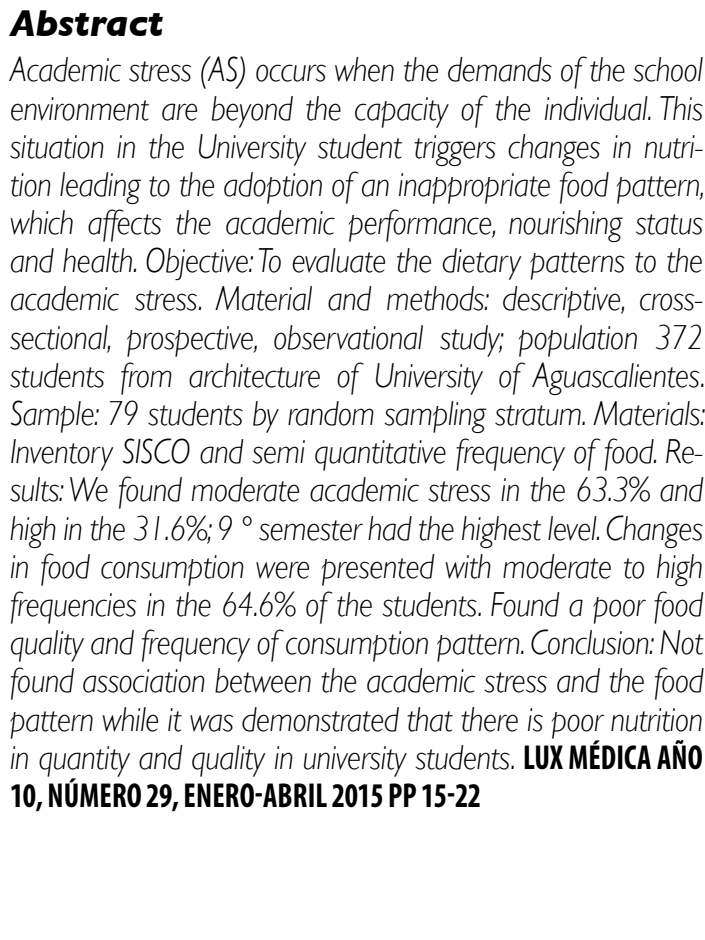 \\
\hline $\begin{array}{l}\text { Palabras clave: Estrés académico, patrón de alime } \\
\text { Arqu }\end{array}$ & ademic stress, eating, architecture, food pattern. \\
\hline
\end{tabular}

* Estudiantes de la Licenciatura en Nutrición del Centro de Ciencias de la Salud de la Universidad Autónoma de Aguascalientes.

** Profesora investigadora de la Licenciatura en Nutrición del Centro de Ciencias de la Salud de la Universidad Autónoma de Aguascalientes.

Fecha de recibido: 27 de enero 2015

Fecha de aceptación: 28 de febrero 2015

Correspondencia: Judith Martín del Campo Cervantes. Departamento de Nutrición y Cultura Física del Centro de Ciencias de la Salud. Universidad Autónoma de Aguascalientes. Avenida Universidad Número 940. Ciudad Universitaria Código Postal 20131. Aguascalientes, Ags., México. Teléfono +52(449)9108443. Correo electrónico jmartic@correo.uaa.mx 


\section{Introducción}

El estrés académico (EA) es un tema poco estudiado y menos aún, relacionarlo a la alimentación de los estudiantes universitarios ${ }^{1}$. El estrés es un proceso que se origina cuando las demandas ambientales superan la capacidad adaptativa de un organismo². El EA es la serie de cambios en los componentes del proceso de "enseñanzaaprendizaje" ${ }^{3}$, producto de la exigente demanda en actividades académicas, debido a las cargas de trabajo ${ }^{4}$, la preocupación en desempeño escolar, el proceso de adaptación al medio universitario, las exigencias educativas y el desarrollo de la vida profesional y personal ${ }^{5}$.

Se ha referido que el $93 \%$ de estudiantes reportan haber tenido algún momento de estrés en el transcurso de la estancia universitaria ${ }^{5}$. En México, Pulido y cols., describen un índice de EA mayor en las carreras de Tecnología y Sistemas, Traducción, Diseño Gráfico y Arquitectura ${ }^{4}$.

La etapa universitaria implica un mayor grado de desafíos, que desencadena la presencia de niveles más altos de estrés, lo que repercute directamente en la adopción de patrones alimentarios deficientes en cuanto a calidad y cantidad de alimentos que se ingieren, que condicionan no sólo en el rendimiento escolar, sino también la calidad de vida y el estado nutricio de la población universitaria1, 6 .

La alimentación del universitario es de baja calidad, se caracteriza por presentar un alto consumo de comida rápida y golosinas, deficiente ingesta de frutas y verduras, poco consumo de agua y consumir alimentos entre comidas. En general la dieta es evaluada como hipercalórica, hiperproteica, deficiente en hidratos de carbono complejos, alta en azúcares simples (50\%) y en grasas (principalmente saturadas y colesterol); además de una importante inactividad física ${ }^{7}$. Contreras Landgrave y cols. ${ }^{8}$, explican que a la mayoría de los universitarios les agradan los alimentos que consumen, y a pesar de saber el poco valor nutritivo de éstos, los consumen por "necesidad", gusto o valor económico. Estas conductas alimentarias son percibidas como condicionadas a la presencia de algún grado de $\mathrm{EA}^{9}$, y no precisamente por falta de conocimiento del tema ${ }^{6}$.

Epidemiológicamente los estudiantes universitarios representan una población adulta joven clave para el desarrollo de actividades de promoción y prevención en materia de salud ${ }^{9}$. Su deficiente alimentación se relaciona con el aumento de la posibilidad de tener sobrepeso, aumento de la grasa corporal y factores de riesgo para enfermedades cardiovasculares ${ }^{10}$.

El patrón alimentario permite conocer la calidad de la dieta en términos cualitativos y cuantitativos, así como para orientar y evaluar intervenciones específicas cuyo objetivo sea modificar comportamientos alimentarios y monitorear su comportamiento en el 
tiempo ${ }^{11}$. En el proceso de la alimentación, se encuentran inmersos factores fisiológicos, sociales y psicológicos ${ }^{12}$; por ello el objetivo del presente trabajo fue evaluar los patrones de alimentación ante el EA.

\section{| | | | | | | | | | | | | | | | | | | | | | | | | | | | | | | | | | | | | | | | | | | | | | | | | | | | | | | | | | | | | | | | | | | | | | | | | | | | | | | | | | | | | | | | | | | | | | | | | | | | | |}

\section{Material y métodos}

Estudio descriptivo, transversal, prospectivo, observacional. La población de estudio fue de 372 alumnos de $1^{\circ}$ a $10^{\circ}$ semestre de la Lic. en Arquitectura de la UAA, de la que se obtuvo una muestra de 79 alumnos por muestreo probabilístico aleatorizado por estratos.

Los criterios de inclusión fueron que pertenecieran a la carrera de Lic. en Arquitectura de la UAA, con edad de 18 a 27 años, que no trabajaran y aceptaran participar en el estudio. Se excluyeron a quienes estuvieran en tratamiento psiquiátrico o con ansiolíticos o bajo un régimen alimentario.

Para evaluar componentes sistémicoprocesuales del EA se utilizó el inventario SISCO del estrés académico, que consta de 31 ítems que permiten determinar: el nivel de intensidad del EA, la frecuencia en que las demandas del entorno son valora- das como estímulos estresores, la frecuencia con que se presentan los síntomas o reacciones al estímulo estresor e identificar la frecuencia de uso de las estrategias de afrontamientos. El cuestionario muestra una confiabilidad por mitades de 0.83 y una confiabilidad en alfa de Cronbach de $0.90^{13}$. Se utilizó una frecuencia semicuantitativa de consumo de alimentos que consta de 9 grupos de alimentos (verduras, lácteos, huevos, carnes y pescados, leguminosas y harinas, frutas, aceites y grasas, dulces y postres, abarrotes y bebidas) con un total 148 alimentos, el instrumento mostró validez con un nivel de significancia para todas las pruebas de $p<0,05^{14}$.

Para el análisis de la información se elaboró una base de datos en el programa Excel 2013 y se utilizó el programa Minitab 17 para calcular el coeficiente de correlación lineal de Spearman.

\section{| | | | | | | | | | | | | | | | | | | | | | | | | | | | | | | | | | | | | | | | | | | | | | | | | | | | | | | | | | | | | | | | | | | | | | | | | | | | | | | | | | | | | | | | | | | | | | | | | | | || ||}

\section{Resultados}

Se estudiaron a 79 alumnos de 1 er a $10 \mathrm{mo}$ semestre de la Licenciatura en Arquitectura de la UAA, de los cuales 42 fueron mujeres $(53.2 \%)$ y 37 hombres (46.8\%), el promedio de edad de la población fue de 20.5 años (DE+1.7 años).

Al evaluar las respuestas del inventario SISCO se identificó la presencia de EA moderado en el $63.3 \%$ de los alumnos, alto en el $31.6 \%$ y bajo o muy bajo en el $5.1 \%$ de la población. En relación a los niveles de intensidad del EA el $50.6 \%$ de la población refiere un estrés moderado, el $21.5 \%$ un estrés alto, el $8 \%$ tiene un nivel muy alto y el $19.9 \%$ reporta niveles bajos y muy bajos de EA. Es importante señalar que las demandas del entorno son valoradas como estresores con una frecuencia de algunas veces hasta siempre en el $46.8 \%$ de los alumnos y el resto de la población refiere una frecuencia nula o casi nula.

Otro de los aspectos que evalúa el inventario SISCO son los síntomas ocasionados por el EA, específicamente la alteración en el consumo de alimentos fue una reacción comportamental que sucedió con frecuencias de moderadas a altas en el $64.6 \%$ de los alumnos.

El semestre que presentó mayor nivel de EA fue el noveno semestre con un nivel de 4.30 en una escala de 5 , seguido del sexto semestre (3.44); tercero, séptimo y octavo refirieron un nivel de 3.40 , el menor 
nivel se encontró en cuarto semestre con 2.30, lo anterior se muestra desglosado en la tabla 1, en donde también se observa que la alteración de consumo alimentario como respuesta al EA, se reportó la mayor alteración en el $3^{\circ}$ semestre y la menor en $1^{\circ}$ semestre.

\section{Tabla I}

Promedios de la presencia, nivel de estrés académico y alteración del consumo alimentario en los estudiantes de Arquitectura de la Universidad Autónoma de Aguascalientes.

\begin{tabular}{|lcccc|}
\hline Semestre & $\mathbf{n}$ & Presencia de EA & Nivel de intensidad del EA & Alteración de consumo alimentario \\
\hline $1^{\circ}$ & 10 & 2.46 & 2.70 & 2.10 \\
\hline $2^{\circ}$ & 9 & 2.57 & 2.66 & 3.00 \\
\hline $3^{\circ}$ & 8 & 3.11 & 3.40 & 3.88 \\
\hline $4^{\circ}$ & 6 & 2.44 & 2.30 & 2.17 \\
\hline $5^{\circ}$ & 9 & 2.62 & 3.00 & 3.00 \\
\hline $6^{\circ}$ & 9 & 2.57 & 3.44 & 3.44 \\
\hline $7^{\circ}$ & 10 & 2.72 & 3.40 & 2.30 \\
\hline $8^{\circ}$ & 5 & 2.74 & 3.40 & 2.20 \\
\hline $9^{\circ}$ & 7 & 3.12 & 4.30 & 3.14 \\
\hline $10^{\circ}$ & 6 & 2.71 & 3.33 & 3.83 \\
\hline
\end{tabular}

$n=79$ alumnos

EA = Estrés académico

Se utilizó una frecuencia alimentaria para valorar el consumo promedio de los diferentes grupos de alimentos, se seleccionaron los grupos y alimentos de mayor relevancia, los cuales se muestran en la figura 1. Como se puede observar, el consumo de frutas y verduras es deficiente, al reportar una ingesta de nunca o casi nunca de un $47.15 \%$ para las frutas y de $52.98 \%$ para las verduras. El $14.6 \%$ de los alumnos consume dulces y postres de 2 a 4 veces por semana. Sobre el consumo de papas y frituras se encontró que el $21.52 \%$ de los alumnos las consume de 2 a 4 veces por semana.

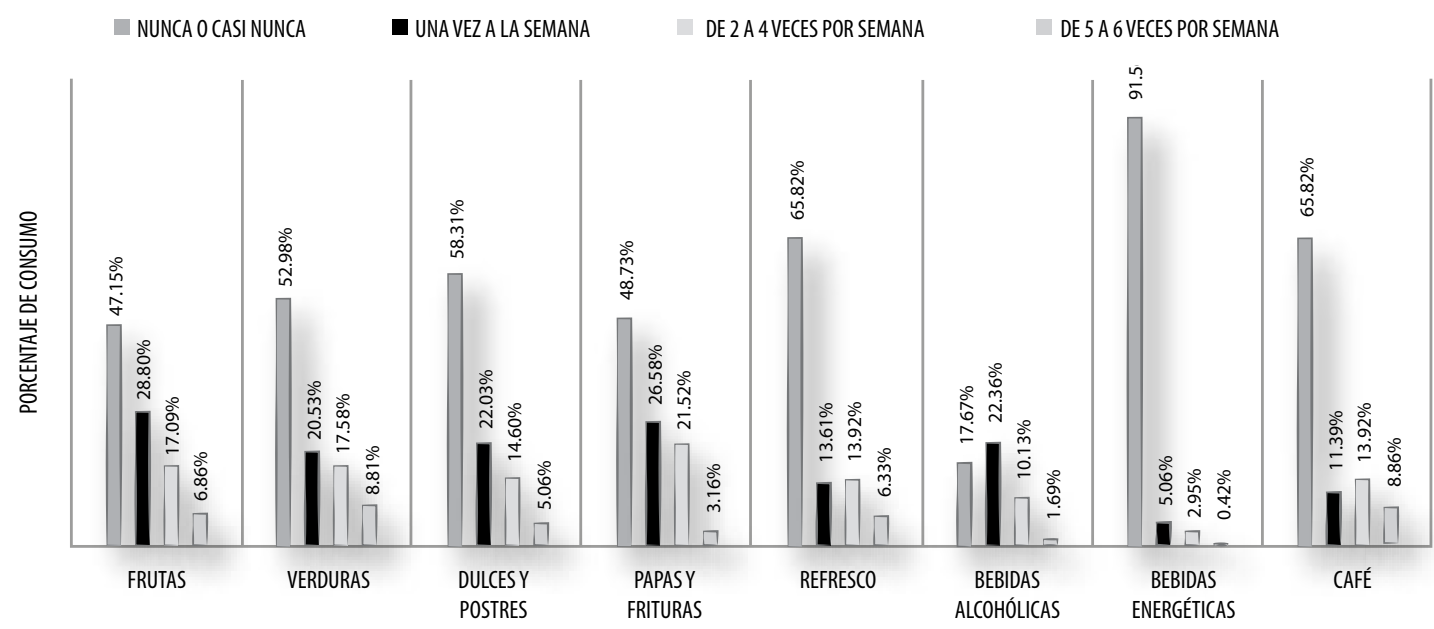

Figura 1. Proporción de frecuencias de consumo de los diferentes grupos de alimentos de los estudiantes de Arquitectura de la Universidad Autónoma de Aguascalientes. $(n=79)$ 
En el análisis de las frecuencias de consumo de alimentos por semestre, se encontró lo siguiente:

El $5^{\circ}$ semestre refirió las frecuencias más altas en el consumo de frutas y verduras de 5 a 6 veces por semana, con una proporción de 15,64\% y 19,44\% respectivamente. Siendo también el semestre que registró mayor consumo de dulces y postres, donde cerca del $20 \%$ los consume de 5 a 6 veces por semana. Se observó que el mayor consumo de alcohol ( $1 \mathrm{vez} \mathrm{a}$ la semana) se presenta en el $6^{\circ}$ semestre, mismo que registró el mayor consumo de refresco por semana, con frecuencias de 2 a 4 veces en el $33.33 \%$ de los alumnos.
En el $8^{\circ}$ semestre se encontró la mayor frecuencia de consumo de papas y frituras de harina, donde el $40 \%$ de los alumnos refieren consumirlas de 2 a 4 veces por semana. En el $9^{\circ}$ semestre se registró el promedio de consumo de café más alto, al ser consumido de 2 a 4 veces por semana por el $43 \%$ de los alumnos, mientras que el $28.57 \%$ lo hace casi a diario.

Se seleccionaron tres semestres representativos de cada nivel de EA, considerando al $4^{\circ}$ como el semestre con bajo nivel de $E A, 6^{\circ}$ semestre con moderado nivel de EA y $9^{\circ}$ semestre alto nivel de $E A$, en los que se realizó el análisis de la frecuencia de consumo que se representa en la figura 2 .

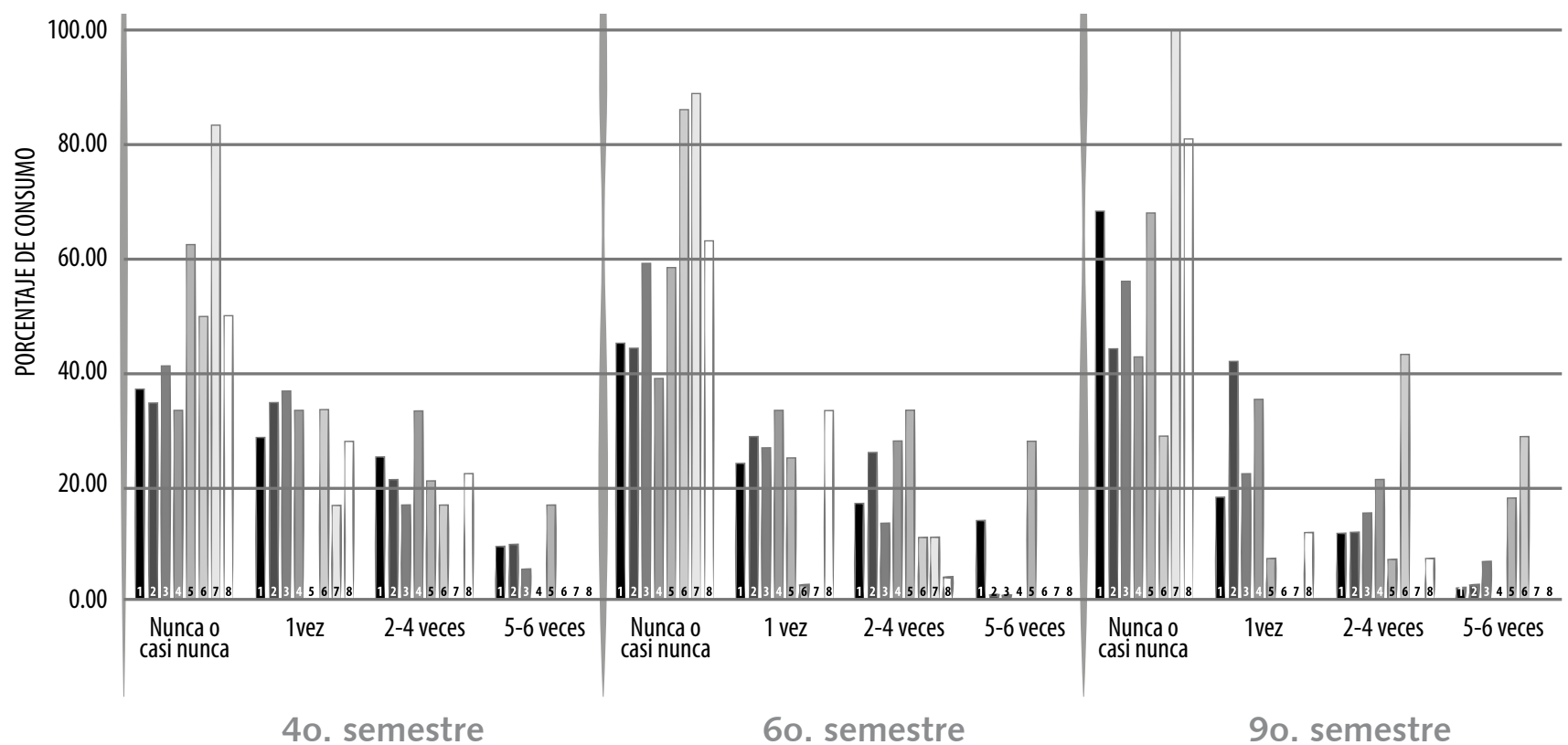

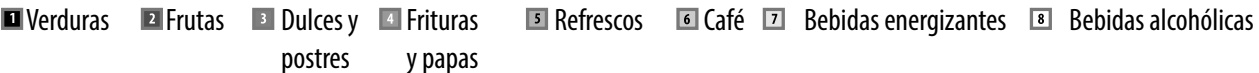
$n=4^{\circ}$ semestre ( 6 alumnos), $6^{\circ}$ semestre (9 alumnos), $9^{\circ}$ semestre ( 7 alumnos)

Figura 2. Frecuencias de consumo alimentario de semestres con nivel de estrés académico leve $\left(4^{\circ}\right)$, moderado $\left(6^{\circ}\right)$ y alto nivel $\left(9^{\circ}\right)$. 
El mayor consumo de verduras se encontró en $6^{\circ}$ semestre, donde el $13.99 \%$ las consume casi a diario. Por otro lado, el consumo de frutas más alto corresponde al $4^{\circ}$ semestre con frecuencias de 5 a 6 veces por semana en el $9.72 \%$ de los alumnos. El consumo de dulces y postres es más frecuente en el $9^{\circ}$ semestre, con un $6.67 \%$ de alumnos que los consume casi a diario. En el $4^{\circ}$ semestre se reportó la ingesta más alta de papas o frituras de harina de 2 a 4 veces por semana que es el $33.33 \%$ de los alumnos, mismo que presenta mayor consumo de bebidas alcohólicas en el $22.22 \%$ de 2 a 4 veces por semana.

Se obtuvo una correlación nula entre las variables nivel de EA y frecuencia de consumo de los diferentes grupos de alimentos, con niveles de significancia $p>0.05$, los valores por grupo se desglosan en la tabla 2.

\section{Tabla 2}

Correlación de estrés académico y frecuencia alimentaria $r=$ coeficiente de correlación, $p=$ nivel de significancia, $E A=$ estrés académico

\begin{tabular}{|lll|}
\hline Grupo de alimentos & $\mathbf{r}$ & $\mathbf{p}$ \\
\hline Verduras & $-0,207$ & 0,519 \\
\hline Frutas & 0,059 & 0,855 \\
\hline Dulces y postres & 0,030 & 0,927 \\
\hline Frituras y papas & 0,060 & 0,853 \\
\hline Refresco & 0,000 & 1,000 \\
\hline Café & $-0,030$ & 0,927 \\
\hline Bebidas energéticas & $-0,099$ & 0,760 \\
\hline Bebidas alcohólicas & $-0,060$ & 0,854 \\
\hline
\end{tabular}

\section{Discusión}

Según la investigación realizada por Feldman y cols ${ }^{15}$ los primeros y últimos semestres de la vida universitaria son un punto crítico para la presencia de EA; en esta investigación se encontró que los niveles de intensidad del EA en los alumnos de la Lic. en Arquitectura varían conforme el cambio de semestre, donde el nivel de intensidad percibido es leve o moderado y va aumentando hasta alcanzar el nivel máximo de EA en $9^{\circ}$ semestre.

Los semestres donde se detecta mayor nivel de EA son $9^{\circ}, 6^{\circ}$, seguido en igual grado por $3^{\circ}, 10^{\circ}$ y $8^{\circ}$ semestre. Lo que difiere del trabajo realizado por Pulido y cols., donde se encontró que los semestres donde se detecta el estrés a mayor grado son $3^{\circ}, 7^{\circ}$ y $8^{\circ}$ independientemente de la carrera que se estudie ${ }^{4}$. En esta investigación se obtuvo una muestra representativa de cada semestre de una sola licenciatura cuyo programa consta de diez semestres en total a diferencia del trabajo de Pulido donde la población fue heterogénea, se analizó el estrés de varias licenciaturas y la mayor parte de la población fue de $1^{\circ}, 3^{\circ}$ y $7^{\circ}$ semestre.

De acuerdo con Alba ${ }^{16}$ se espera que la presencia del estrés en los alumnos universitarios se atenúe con el paso del tiempo, sin embargo en el presente trabajo se describe que la frecuencia con que aparece el EA es variante de acuerdo al semestre que se esté cursando, lo que se debe a las diferentes exigencias que implica cada semestre en relación al tipo y número de materias, trabajos, exámenes, entre otros. 
En el trabajo de Cervera ${ }^{7}$ se encontró que el deficiente consumo de frutas y verduras, y por otro lado el alto consumo de comida chatarra y golosinas, describen el patrón alimentario de los estudiantes universitarios; al evaluar la frecuencia alimentaria de este grupo de alimentos en nuestra población se encontró un deficiente consumo de los primeros alimentos y una frecuencia de consumo regular de refrescos, dulces, postres y frituras o papas fritas, lo que coincide con lo referido por Cervera, esto puede condicionar el aumento de la posibilidad de tener sobrepeso, aumento de la grasa corporal y factores de riesgo para enfermedades crónico degenerativas ${ }^{10}$.

Troncoso y cols ${ }^{9}$ muestran que el estrés académico se identifica como un factor influyente en la modificación de los patrones alimentarios en cuanto a calidad y cantidad de los alimentos que se ingieren ${ }^{12}$; sin embargo, esta asociación no pudo ser demostrada estadísticamente en nuestra población, probablemente debido a discrepancias en las respuestas de la frecuencia alimentaria al no ser contestadas adecuadamente, esto por falta de atención, interés o el poco tiempo dedicado para la contestación del cuestionario.

En este trabajo se encontró que los alumnos perciben un cambio en su alimentación condicionada por la presencia del EA evidencia que concuerda con Troncoso y $\operatorname{cols}^{12}$, donde demuestra que el estrés desencadena la modificación del patrón alimentario en cuanto a calidad y cantidad de alimentos.

Para la realización de estudios posteriores respecto a este tema, se debe considerar la aplicación de una frecuencia alimentaria enfocada a aquella alteración en el consumo de alimentos que se consideran de mayor relevancia ante la presencia de estrés académico, como frutas y verduras, snaks, comida rápida, golosinas y bebidas azucaradas ${ }^{7,16}$; así como considerar extensión del cuestionario y el tiempo disponible para realizarlo, el periodo en el que se recolecta la información, además de contrastar periodos de mayor o menor EA.

Los resultados reflejan una inadecuada frecuencia alimentaria y por lo tanto un patrón alimentario alterado, lo que ocasionan una reducción en la calidad de la dieta y por lo tanto de la salud ${ }^{17}$.

\section{Conclusiones}

No encontramos una asociación entre el EA y la modificación del patrón de alimentación en los estudiantes de la Lic. en Arquitectura de la UAA, lo que sugiere que existen más factores que influyen en una incorrecta alimentación.

\section{Bibliografía}

1. Martín M. Estrés académico en estudiantes universitarios. Apuntes de psicología. 2007; 25: 87-99.

2. Lahuerta B, Sainz S, Sánchez A, Matachana M. Promoción de la salud y apoyo psicológico al paciente. 4ta ed. Madrid, McGraw-Hill Interamericana de España, 2011, pág. 52-54

3. Román $C$, Hernández Y. El estrés académico: una revisión crítica del concepto desde las ciencias de la educación. Revista Electrónica de Psicología Iztacala. 2011; 14: 1-14.

4. Pulido $R$, Serrano $S$, Valdés $C$, Chávez $M$, Hidalgo $M$, Vera $G$. Estrés académico en estudiantes universitarios. Psicología y salud. 2011; 21: 31-33.

5. Valenzuela L, Fraijo B. Integración y Estrés en Estudiantes Universitarios. XI Congreso Nacional de Investigación Educativa. 2009; 16: 1-7. 
6. Barreto J, Augusta M. Estrés y recursos de enfrentamiento del cuidador. Salud mental. 2011; 34: 131 132.

7. Cervera B, Serrano U, Cruz V, Milla T, García M Hábitos alimentarios y evaluación nutricional en una población universitaria. Nutr Hosp. 2013; 28: 438446.

8. Landgrave C, Camacho R, Ibarra E, López G, Escoto $P$, Pereira $A$, et al. Los hábitos alimentarios de estudiantes universitarios. Revista digital universitaria. 2013; 14: 1-15.

9. Troncoso P, Amaya P. Factores sociales en las conductas alimentarias de estudiantes universitarios. Rev Chil Nutr. 2009; 36: 1090-1097.

10. Espinoza O, Rodríguez R, Gálvez C, MacMillan K. Hábitos de alimentación y actividad física en estudiantes universitarios. Rev Chil Nutr. 2011; 38: 458465.

11. Herrán O. Calidad de la dieta de la población adulta en Bucaramanga y su patrón alimentario. Colombia Médica. 2005; 36: 94-102.

12. Troncoso PC. Percepción de la alimentación durante la etapa de formación universitaria. Chile. Rev Costar
Salud Pública. 2011; 20: 83-89.

13. Barraza M. El inventario SISCO del estrés académico. Universidad pedagógica de Durango. 2007; 89-93.

14. Monsalve A, González Z. Diseño de un cuestionario de frecuencia para evaluar ingesta alimentaria en la Universidad de Antioquia, Colombia. Nutr Hosp. 2011; 26:1333-1344.

15. Feldman L, Goncavalves L, Chacón-Puignau G, Zaragoza J, Bagés N, De Pablo J. Relaciones entre estrés académico, apoyo social, salud mental y rendimiento académico en estudiantes universitarios venezolanos. Univ. Psichol. 2008; 7: 739-751.

16. Alba D, Arenas A, Botero F. Estrés, estrategias de afrontamiento, estilos de aprendizaje, y comunicación asertiva, en una muestra de estudiantes del Programa de Medicina de la Universidad de Manizales, y diseño de un proceso de mejora 2012. Universidad de Manizales. 2013; 13: 189-190.

17. Alejo Z, Rodríguez A, López D, Almaguer P. Patrón alimentario en adolescentes de octavo grado y su repercusión en la adecuación dietética. Revista Electrónica de las Ciencias Médicas en Cienfuegos. 2011; 9 : 518-522. 